\title{
Nicht aktualisierte Leitlinien werden von der AWMF nicht mehr publiziert
}

\section{Zusammenfassung}

Nach dem Beschluss der ständigen Leitlinienkommission der AWMF werden zukünftig die Leitlinien, deren Gültigkeit abgelaufen ist, von der AWMF nicht mehr im Internet publiziert. Als Ablaufdatum gilt die Angabe der Fachgesellschaft(en), wann die Leitlinie turnusgemäß überprüft werden soll - falls die Fachgesellschaft(en) dazu keine Angaben gemacht hat, werden die Leitlinien spätestens 5 Jahre nach ihrer Erstellung von der AWMF als "nicht aktualisiert" eingestuft und aus dem Publikationssystem entfernt.

\author{
Wolfgang Müller \\ 1 AWMF, Düsseldorf
}

\section{Text}

Bisher wurden diese nicht aktualisierten Leitlinien mit einer roten Marke gekennzeichnet, in ein separates Verzeichnis "Nicht aktualisierte Leitlinien" verschoben und im internen Stichwort-Suchsystem der AWMF-Leitlinien nicht mehr nachgewiesen. Sie waren aber weiterhin über das Internet verfügbar und konnten mit externen Suchmaschinen (Google etc.) gefunden werden. Zum Oktober 2008 wird dieses Verzeichnis komplett gelöscht.

Die Fachgesellschaften sind aufgefordert, ihre Aktualisierungstermine einzuhalten und der AWMF diese Aktualisierungsrunden durch eine Anmeldung bekannt zu geben. Die AWMF-Geschäftsstelle weist die Fachgesellschaften mit einem Formschreiben ca. 6 Monate vor dem Termin auf das bevorstehende Auslaufen der Leitlinien hin. Wenn die Fachgesellschaft keine Aktualisierung anmeldet oder keine aktualisierte Leitlinie zur Publikation einreicht, wird die bisherige Leitlinien-Datei nach Ablauf der Frist gelöscht.

Wir weisen auch darauf hin, dass die Fachgesellschaften als Ersteller und Herausgeber der Leitlinien aus Dokumentationsgründen auch die nicht aktualisierten Versionen speichern und verfügbar halten sollten. Die AWMF publiziert jeweils nur die aktuell gültigen Leitlinien und verweist Anfragen nach früher gültigen Leitlinien immer an die Fachgesellschaften, von denen diese Leitlinien herausgegeben wurden.

\section{Korrespondenzadresse:}

M.A. Wolfgang Müller

AWMF-Geschäftsstelle, Ubierstr. 20, 40223 Düsseldorf office@awmf.org

\section{Bitte zitieren als}

Müller W. Nicht aktualisierte Leitlinien werden von der AWMF nicht mehr publiziert. GMS Mitt AWMF. 2008;5:Doc22.

Artikel online frei zugänglich unter

http://www.egms.de/en/journals/awmf/2008-5/awmf000165.shtml

\section{Eingereicht: 23.09 .2008}

Veröffentlicht: 23.09.2008

\section{Copyright}

(C)2008 Müller. Dieser Artikel ist ein Open Access-Artikel und steht unter den Creative Commons Lizenzbedingungen

(http://creativecommons.org/licenses/by-nc-nd/3.0/deed.de). Er darf vervielfältigt, verbreitet und öffentlich zugänglich gemacht werden, vorausgesetzt dass Autor und Quelle genannt werden. 